\title{
eJRIEPS
}

Ejournal de la recherche sur l'intervention en éducation physique et sport

$45 \mid 2019$

Varia

\section{Quel développement professionnel pour des enseignants associés dans une formation de type recherche-action participative?}

Claire Mattei-Mieusset, Geneviève Emond et Pierre Boudreau

\section{OpenEdition}

Journals

Édition électronique

URL : http://journals.openedition.org/ejrieps/635

DOI : $10.4000 /$ ejrieps. 635

ISSN : 2105-0821

Éditeur

ELLIADD

Référence électronique

Claire Mattei-Mieusset, Geneviève Emond et Pierre Boudreau, « Quel développement professionnel pour des enseignants associés dans une formation de type recherche-action participative? », eJRIEPS [En ligne], 45 | 2019, mis en ligne le 01 avril 2019, consulté le 01 août 2019. URL : http:// journals.openedition.org/ejrieps/635; DOI : 10.4000/ejrieps.635

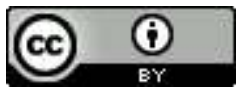

La revue eJRIEPS est mise à disposition selon les termes de la Creative Commons Attribution 4.0 International License. 
Quel développement professionnel pour des enseignants associés dans une formation de type recherche-action participative ?

Claire Mattei-Mieusset ${ }^{*}$, Geneviève Emond ${ }^{* *}$ \& Pierre Boudreau ${ }^{* * *}$

* Université de Reims Champagne Ardenne, France.

** Université du Québec à Montréal, Canada.

*** Université d'Ottawa, Canada.

Résumé

Cet article est issu d'une recherche internationale : PReParE-Eps, 2015-2018 (Belgique, Canada, États-Unis, France, Suisse), portant sur le développement des compétences des enseignants associés (EA) en Education Physique et Sportive (EPS), dans leur fonction d'accompagnement (Paul, 2009) d'un enseignant débutant. En contexte français, cinq EA bénéficient d'une formation de type "recherche-action participative " (RAP) (Chevalier et Buckles, 2013). La RAP, utilisée dans un but de développement professionnel, engage les participants dans toutes les étapes d'une telle formation tout en tenant compte des compétences déjà acquises et de leur autonomie comme professionnel. Les données se présentent sous la forme de discours oral et écrit des EA, en amont, pendant, et en aval du dispositif organisé en quatre journées réparties sur cinq mois. Nous caractérisons le processus de développement professionnel (Grosstephan, 2010 ; Jorro, 2014) de deux EA à partir de trois indicateurs : réflexivité, mobilisation d'outils conceptuels, construction d'un genre professionnel (Clot, 1999), dans quatre phases : insécurité, expérimentation, appropriation, consolidation (Boucher et Jenkins, 2004), tout en cernant les apports spécifiques d'une RAP dans cette dynamique.

Mots-clés : formation ; accompagnement ; enseignants-associés (EA); recherche-action participative (RAP) ; développement professionnel ; Education Physique et Sportive (EPS)

\section{Introduction}

Cette contribution est issue d'une recherche internationale : PReParE-Eps, 2015-2018 (Belgique, Canada, États-Unis, France, Suisse) portant sur le processus de construction et de perfectionnement des compétences des enseignants associés (EA) en Education Physique et Sportive (EPS), dans leur fonction d'accompagnement (Mattéï-Mieusset, 2013 


\section{eJRIEPS 45 (Avril 2019)}

; Paul, 2009) d'un enseignant débutant. En contexte français, nous avons offert à cinq $E A$ une formation de type « recherche-action participative » (RAP) (Chevalier et Buckles, 2013). Dans ce texte, nous caractérisons le processus de développement professionnel (Grosstephan, 2010 ; Jorro, 2014) de deux EA, tout en cernant les apports spécifiques d'une RAP à cette dynamique.

\section{Contexte}

La pratique de l'enseignement en situation réelle, sous la forme de stages supervisés en milieu scolaire, est considérée comme un moyen incontournable de développer des compétences professionnelles. Elle permet d'assurer une formation de qualité aux futurs enseignants (Desbiens et al., 2009, 2013 ; Molina et Gervais, 2008). Dans ce contexte, l'EA se voit confier l'accompagnement d'un débutant dans son activité d'enseignant. Cette mission implique de multiples tâches telles qu'accueillir le stagiaire, l'aider dans son travail de conception de l'enseignement, l'observer et l'aider à réguler sa pratique. L'EA est également responsable de l'évaluation des compétences professionnelles de ce futur enseignant. Plusieurs auteurs (Boutet et Pharand, 2008 ; Chaliès et Durand, 2000) s'accordent pour dire que le rôle d'EA est différent, quoique complémentaire, à celui d'enseignant. II ne suffit pas de savoir enseigner pour savoir accompagner un débutant. Ce travail d'accompagnement en formation requiert la maîtrise de compétences, dans l'activité d'enseignement de l'EPS, dans la formation à cette activité et dans la gestion de la relation avec le débutant. L'EA doit pouvoir adapter son interaction, en tenant compte, des besoins, des connaissances et des motivations du stagiaire, de ses propres connaissances et du contexte scolaire. La proximité des fonctions d'enseignant et d'EA peut aussi générer des dilemmes (Mattéi-Mieusset et Brau-Antony, 2016).

Ces constats aiguisent l'intérêt envers la formation des EA. De nombreux auteurs (Altet et al., 2002 ; Boutet et Villemin, 2014 ; Carlier, 2009) soulignent le manque de recherches sur ces formations. Dans une recension des quelques formations des EA en EPS (13), Boudreau (2009) note la prédominance d'un modèle de formation de type " applicationniste » souvent critiqué, entre autres par St-Arnaud (2003), pour « son manque de prise en considération de l'idiosyncrasie de la situation réelle et des connaissances de l'intervenant impliqué» (p.131). Tentant de progresser dans ces formations, nous avons choisi une approche de formation de type RAP, une recherche-formation «avec » et non «sur » les EA. 


\section{eJRIEPS 45 (Avril 2019)}

\section{Cadre conceptuel}

Les pratiques d'accompagnement prennent toute leur place dans le contexte de nouvelles modalités de professionnalisation beaucoup plus individualisées et singulières (Roquet, 2009), privilégiant la construction de l'autonomie des personnes dans leur développement professionnel. La RAP permet cette construction, en jouant le rôle de construit théorique et de méthodologie de formation.

3. 1. La « recherche-action participative »

La RAP (Chevalier et Buckles, 2013) a une visée heuristique et pragmatique. Heuristique, parce qu'elle permet une régulation flexible et constante d'éléments de contenus et organisationnels du travail collaboratif. Pragmatique par son engagement en vue de trouver des solutions à une problématique partagée par tous sur des objets à la fois de formation et de recherche. Elle intègre une réflexion collaborative à l'intérieur de ce qui est appelé des « systèmes apprenants », au sein desquels agissent en synergie complexe : la pensée, par les connaissances issues de la recherche ; l'expérience, par l'action-réflexion sur l'action ; la société, par la participation collective (ibid., p.3). Ainsi, les formateurs-chercheurs praticiens de la RAP accompagnent de manière inductive les changements sociaux à l'aide de leurs savoirs et de leur savoir accompagner. Ils s'appuient tant sur une science de l'action (Dewey, 1938) que sur les théories psychosociologiques (dans la lignée de Rogers, 1970). La recherche permet d'accéder avec les praticiens à des savoirs et à des connaissances construites dans l'action, par le biais de leur expérience et de la transformation de leur activité. Elle est participative par son impact sur et son intégration à la vie en société démocratique (Chevalier et Buckles., 2013, p.10), mise en œuvre dans la gestion du groupe. Chaque être humain construit son savoir et dans la très grande majorité des cas, le construit en interaction avec d'autres humains, dans son quotidien personnel et professionnel et non dans un monde hypothétique et surtout parcellaire comme celui de la science positiviste. C'est ce qui amène Chevalier et Buckles (2013) à écrire : «Dans une perspective plus large, la science est une audacieuse incursion dans le domaine des multiples modalités de construction du savoir. » (p.3) pour bien illustrer que la science ne peut être réduite à une approche positiviste de la recherche. Les résultats présentés dans notre étude cherchent justement à décrire comment deux des participants à notre projet ont construit leur savoir en prise directe avec leur travail d'accompagnement.

Selon ces auteurs, dans une RAP, la recherche est vue comme un exercice de communication chercheur-participant et de création de savoir intégré à la vie publique (dans notre projet à un programme de formation à l'accompagnement ). La RAP cherche à faire 


\section{eJRIEPS 45 (Avril 2019)}

vivre des activités de recherche qui engagent les participants dans ce qui fait sens pour eux dans leur fonction d'accompagnement. Dans cette orientation pragmatique, les personnes engagés dans une RAP, autant chercheurs-formateurs que participants, planifient et développent des moyens habiles pour comprendre leur présent, donner forme à leur propre futur et devenir les co-auteurs de leur propre cheminement.

Au sein de la RAP, le chercheur-formateur agit avec un double statut. En tant que chercheur, il produit des connaissances sur l'objet de formation. II s'appuie entre autres alors sur une méthodologie de recherche qui inclut des artefacts participatifs, telle que l'exercice de la roue socratique, un outil schématique de priorisation et d'évaluation (Chevalier et al., 2013, p.47). En tant que formateur, à partir de l'identification d'activités spécifiques à la fonction d’EA, problématisées et constamment réévaluée avec le groupe, il accompagne les praticiens en formation, individuellement et collectivement, dans leur développement professionnel. Nous expliciterons plus bas dans la section méthodologie comment ce double statut s'est concrétisé dans notre projet.

\section{2. L'accompagnement en formation des enseignants}

L'objet de notre RAP est le développement des EA dans leur pratique d'accompagnement d'un enseignant-débutant. Paul (2009) définit l'accompagnement comme un mode d'aide dans lequel une personne se joint à une autre en adoptant une posture de secondarité (en retrait pour valoriser celui qui est accompagné et non pour mettre sa propre expertise en valeur), pour l'aider à changer d'état. Cette transformation de l'accompagné ne peut exister sans une demande de sa part. Ces deux personnes cheminent ensemble et le but, la durée, l'organisation peuvent évoluer en fonction de l'accompagné. Ce faire ensemble implique les deux personnes, ne pouvant laisser indemne l'accompagnateur qui donne et se laisse prendre, dans ce moment de transition temporaire et singulier. L'accompagnateur prend ainsi en compte les besoins et le but de l'accompagné. Paul précise que le contexte de mise en œuvre oriente fondamentalement l'activité des acteurs. Ainsi ici, le contexte de la formation des enseignants en EPS impose des contraintes sur l'activité d'accompagnement de l'EA jusqu'à être sources de difficultés. Par exemple, la dyade ne se choisit pas pour cheminer ensemble. L'institution demande à l'EA d'adopter ce que Donnay (2008) appelle une attitude de compagnon réflexif afin d'amener le débutant à regarder sa pratique le plus rigoureusement et objectivement possible et l'aider à en faire une analyse pour pouvoir la questionner et la faire évoluer. Enfin, le contexte du stage en situation réelle avec des élèves demande un certain degré de performance dans la qualité de l'enseignement et fait naitre des dilemmes chez l'EA comme celui d'« intervenir, faire pour le débutant ou s'effacer, le 


\section{eJRIEPS 45 (Avril 2019)}

laisser faire seul » (Mattéï-Mieusset et Brau-Antony, 2016), lorsqu'il l'observe dans sa pratique d'enseignement. Ces constats révèlent la spécificité de cet accompagnement en formation et la nécessaire construction de compétences pour les EA.

\section{3. Le processus de développement professionnel et ses indicateurs}

Le développement professionnel « englobe la construction de compétences lors de formations individuelles ou collectives, mais aussi la construction de compétences nouvelles par la pratique et la réflexion sur la pratique, ainsi que les transformations identitaires des individus ou des groupes » (Lefeuvre, Garcia et Namolovan, 2009, p.157). Notre focale est celle de l'acteur qui élargit, enrichit, approfondit, en signification et en activité, son rapport à lui-même, à autrui et au monde (Jorro, 2014), dans une dynamique d'engagement dans un collectif professionnel « apprenant », très similaire au « système apprenant » de la RAP. II s'agit d'identifier la manière, dont le sujet s'organise et structure son activité de développement et comment cette activité le structure en retour (ibid.). Pour ce faire, nous prenons en compte trois indicateurs de développement professionnel : la réflexivité, la mobilisation d'outils conceptuels (Grosstephan, 2010), ainsi que la construction d'un genre professionnel (Clot, 1999).

\subsubsection{La réflexivité}

La mise au jour de ce qui se joue dans son activité d'accompagnement amorce la réflexion de l'EA sur les liens entre les visées, les moyens et les résultats de son action. Elle lui permet ainsi de pointer les ressources présentes ou manquantes de son activité (Clot, 1999) et de questionner le sens de son agir pour mieux l'objectiver. Cette objectivation lui permet de s'extraire de la situation singulière pour se projeter dans d'autres moments de son travail avec d'autres acteurs, stagiaires ou collègues. II met ainsi en œuvre une activité cognitive de généralisation (Grosstephan, 2010) qui peut augmenter son pouvoir d'action.

\subsubsection{La mobilisation d'outils conceptuels}

Les ressources conceptuelles, telles que des modèles d'analyse d'une situation d'enseignement, des outils ou dispositifs issus de travaux de recherche, sont mobilisés, discutés en contexte de formation et permettent à l'EA d'expliciter de façon objectivée et distanciée son activité dans différentes situations d'accompagnement. Au retour en contexte professionnel, l'EA choisit d'inclure singulièrement certaines de ces ressources dans sa pratique. La présentation au groupe de leur utilisation par le praticien, dans ses intentions et la réalisation en contexte d'accompagnement du débutant, construit de nouvelles connaissances. Ceci permet d'observer un développement de nouvelles compétences.

\subsubsection{La construction d'un genre professionnel}




\section{eJRIEPS 45 (Avril 2019)}

La reconnaissance par les EA, de compétences, ressources, postures, gestes professionnels, difficultés, dilemmes, communs à tous et spécifiques de la fonction, construit un répertoire d'appartenance à un collectif. Un sentiment d'appartenance à un genre professionnel se développe, même si chacun se singularise et se différencie par son style (Clot, 1999). Les EA construisent et approfondissent une identité différente et complémentaire de celle d'enseignant. La présence de ces indicateurs témoigne d'un développement professionnel vu comme un processus dans lequel Boucher et Jenkins (2004) identifient quatre phases : une « phase d'insécurité » devant les changements à venir, une « phase d'expérimentation » de nouveaux outils, une « phase d'appropriation » et enfin, une «phase de consolidation » qui stabilise la pertinence de la nouvelle activité.

\section{4. Question de recherche}

Tout cela mène à notre question de recherche : quelles sont les caractéristiques du processus de développement professionnel d'un EA dans sa pratique d'accompagnement d'un enseignant d'EPS, par la médiation d'une RAP ?

\section{Méthodologie}

En contexte français, la recherche-formation se déroule dans l'académie de Reims Champagne-Ardenne en collaboration entre l'université (le laboratoire CEREP : Centre d'Etude et de Recherche sur les Emplois et la Professionnalisation et l'ESPE : Ecole Supérieur du Professorat et de l'Education) et le Rectorat. Avec l'accord de leur hiérarchie, nous sollicitons par mail une liste d'EA en EPS, enseignants en lycée. Cinq personnes se portent volontaires et bénéficient de quatre journées (J1 à J4) de RAP de 6h, réparties sur cinq mois en 2016. Les dates de chaque session sont définies en accord avec les participants. Trois enseignants assument les fonctions d'EA en 2016. Les deux derniers ont tenu ce rôle les années précédentes.

\section{1. Description du dispositif RAP}

Le jour 1 (J1) ( $1^{\text {er }}$ février 2016) a pour objet de, créer une relation et un climat de confiance entre les participants, s'approprier les objectifs du projet de recherche-formation, contractualiser les modalités de travail. Chaque EA prend la parole pour dire au collectif comment il se situe à partir de trois critères proposés par le chercheur-formateur : l'engagement dans la RAP, le processus de développement et la répercussion possible sur l'activité d'enseignement et d'EA. Ce rituel est proposé au début et en fin de chaque journée de formation. Le travail se poursuit en construisant un espace d'intéressement commun sur les compétences liées à la fonction d'accompagnement d'un enseignant débutant en EPS. 


\section{eJRIEPS 45 (Avril 2019)}

Chacun des participants se positionne en début de $\mathrm{J} 1$ et envisage un objectif de développement professionnel dans cinq compétences choisies, à l'aide d'un outil : l'exercice de la roue socratique (Chevalier et al., 2013, p.47) repris à la fin de la quatrième journée. Le groupe co-construit l'objet précis de recherche-formation : «l'activité d'observation du débutant en situation d'enseignement, en vue d'une régulation. Quoi et comment observer, dans quel but ? ». Le chercheur-formateur explicite le parti pris de lier tout au long de la RAP, l'expérience et la pratique des EA avec des outils et connaissances issus de la recherche, au service de la formation et de la recherche. II propose de recueillir des traces vidéo de la pratique de deux EA dans l'inter-session dans un but d'analyse en groupe. Elsa et Thom se portent volontaires.

Le jour 2 (J2) (15 mars 2016) permet d'engager le travail d'analyse de la pratique de ces deux praticiens dans leur activité d'observation d'un étudiant de Licence-3 pour Thom et d'un étudiant de Master 2 pour Elsa. L'entretien d'auto-confrontation croisée (EACC) met chacun des deux successivement devant les images de son activité à propos de moments semblables (ex : l'EA observe le débutant donnant les consignes pour engager les élèves dans la tâche). Avec l'aide du chercheur-formateur, chacun explicite son activité en présence des autres EA qui observent et notent pour mémoire ce sur quoi ils sont d'accord, ce qu'ils font différemment et ce qui les questionne. Les commentaires qui suivent, des trois autres EA dont le collègue également filmé, font émerger des controverses professionnelles discutées autour de gestes du métier identifiés par le groupe et des différences repérées dans les deux pratiques filmées. L'analyse entre pairs et avec le chercheur-formateur peut alors s'affiner dans la compréhension des mobiles, des destinataires, des buts poursuivis en lien avec l'efficience de l'activité d'accompagnement et les effets sur le débutant. Des apports théoriques sur le concept d'activité et les méthodes d'AC (Clot, 1999) sont alors mobilisés, de même que des connaissances sur une modélisation de l'activité enseignante (Bucheton et Soulé, 2009), afin de soutenir et structurer l'analyse en collectif. En fin de journée, Thom et Elsa décident de s'emparer d'éléments travaillés dans ce J1 au service de leur pratique d'observation, dans l'inter-session.

Le jour 3 (J3) (22 avril 2016) poursuit le travail d'analyse de l'activité d'observation de chacun des deux EA, à partir du choix d'autres séquences des vidéos d'Elsa et de Thom. Cela permet d'approfondir la réflexion sur la posture des EA dans leur observation, les dilemmes et les difficultés associés, le choix de ce qu'ils observent et avec quel(s) outil(s), les effets sur le débutant. Suit une analyse structurée de la production de la trace d'une activité d'observation et de régulation menée et explicitée par Thom. Un des deux 


\section{eJRIEPS 45 (Avril 2019)}

chercheurs de l'équipe internationale (par ailleurs en appui pour le recueil de données, les échanges post et ante journées de RAP avec le chercheur-formateur) apporte également en visioconférence, des connaissances complémentaires sur l'observation ciblée d'une pratique d'enseignement. Le groupe négocie en fin de journée l'objet de formation du J4 : l'activité de régulation qui suit l'observation.

Lors du jour 4 (J4) (13 juin 2016), le travail s'engage sur l'activité d'entretien de régulation à partir de la suite de l'étude de cas présentée par Thom, puis d'un jeu de rôle mettant en valeur des postures de stagiaires et leurs effets sur celle des EA. Pour suivre, est abordée l'analyse d'un outil d'évaluation de construction de compétences professionnelles d'un débutant, construit et utilisé par Thom avec son stagiaire : une roue socratique transformée (fig. 1), enrichie d'une mutualisation d'expériences des autres participants.

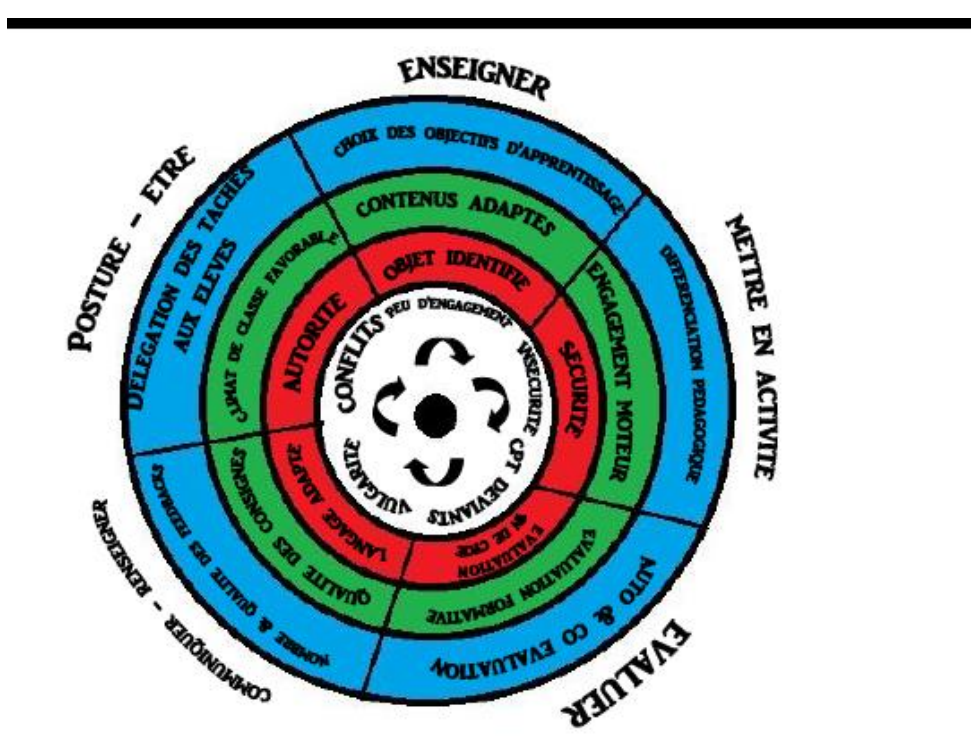

Figure 1. Production de la roue socratique pour l'évaluation d'un stagiaire, présentée par Thom aux autres participants au J4

La dernière partie de la journée est consacrée à une partie de l'évaluation du dispositif RAP. Chaque EA complète sa roue socratique établie en début de recherche-formation en répondant aux consignes suivantes : "aujourd'hui, je me situe où dans les cinq compétences ? » et «si je m'auto-évaluais aujourd'hui au J1, je me situerais où ? ». Au bilan oral rituel individuel s'ajoute, pour chacun, des perspectives de formations éventuelles et l'explicitation au collectif de son positionnement dans la roue socratique (figs. 2 et 3 ). 


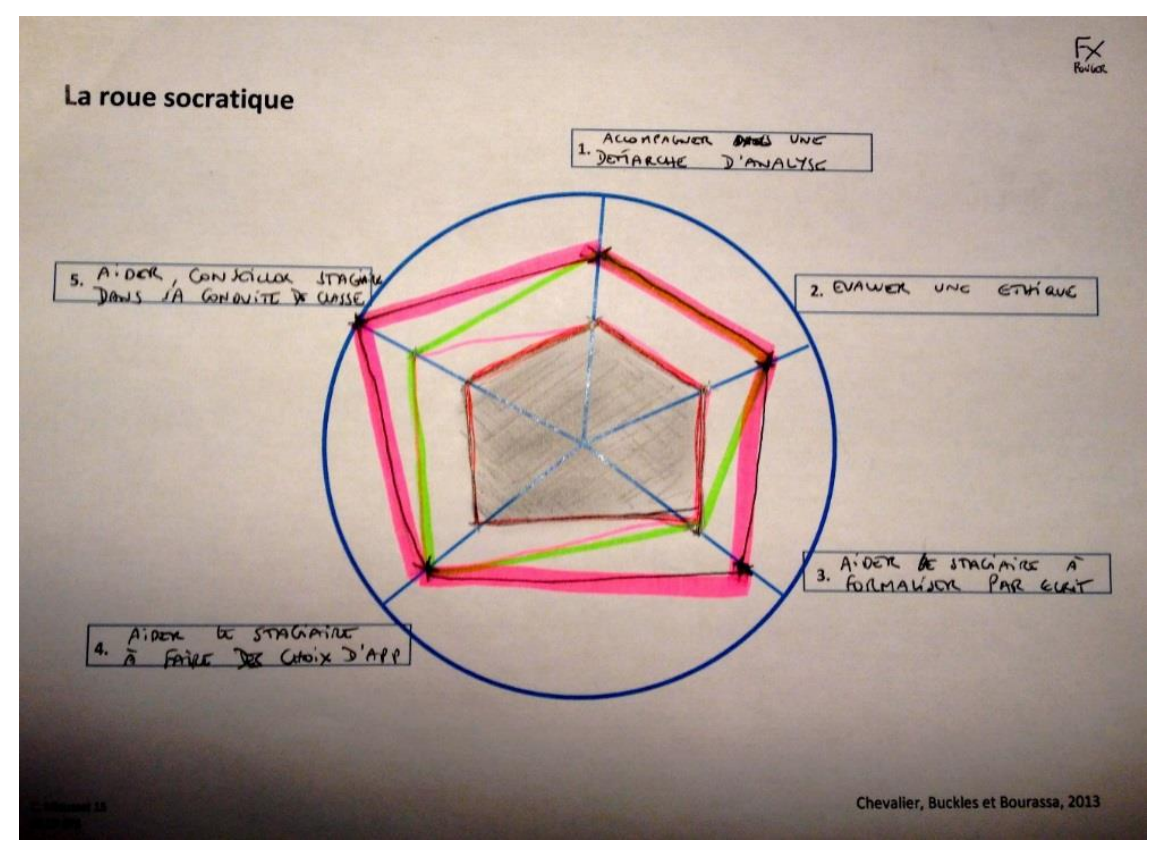

Figure 2. Exercice de la roue socratique (Thom en début puis en fin de formation)

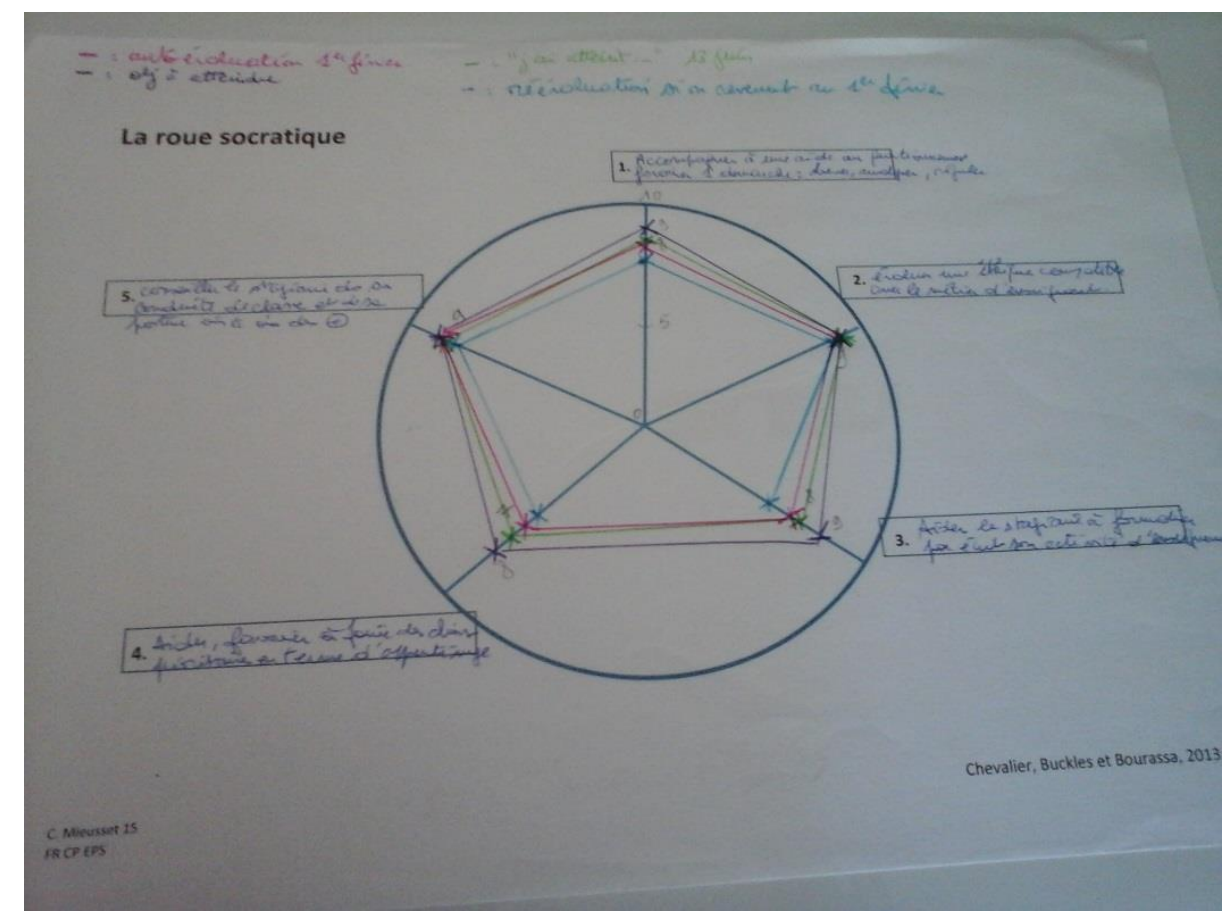

Figure 3. Exercice de la roue socratique (Elsa, en début et en fin de formation)

A la suite de la formation chaque EA est invité à envoyer par mail au chercheur-formateur un écrit réflexif portant sur ce que cette RAP a contribué à développer pour lui et à quels outils ou moments de la RAP, il attribue ces transformations. Le concept de RAP mis de l'avant par Chevalier et Buckles (2013) et utilisé dans notre recherche implique une planification continue des activités de formation et de recherche tout au long du projet pour 


\section{eJRIEPS 45 (Avril 2019)}

prendre en considération les besoins évolutifs de formation, le cheminement professionnel des EA d'une session à l'autre, sans mettre de côté l'objectif de recherche et les données à recueillir. Si les jours 1 à 4 étaient les moments forts de la formation, la planification continue, sous forme de deux rencontres entre le chercheur-formateur et deux autres membres du groupe de recherche, a servi à faire un bilan de la journée de formation vécue (debriefing) et des données de recherche recueillies. Ces rencontres en trio ont permis également de mettre de l'avant des propositions d'activités de formation et des données à recueillir pour la journée suivante, de discuter de leur pertinence tant sur le plan de la recherche que de la formation. Cette stratégie de partage de connaissances et d'expertise entre chaque session a enrichi la démarche de RAP et permis de continuellement avoir à l'esprit les deux volets de formation et de recherche, de même qu'à réaliser ce que nous appellerions un «pré-analyse » des données.

\section{2. Recueil de données}

Nous recueillons des données sous la forme de discours oral ou écrit de type réflexif des EA dans différents cadres, par le biais : d'entretiens individuels pré-formation (janvier 2016) avec un chercheur, qui visaient à dévoiler la trajectoire et les représentation de chacun sur leurs deux pratiques : enseignant d'EPS et EA; de prises de parole individuelles en collectif, au début et à la fin de chaque journée ; des EACC (J2 et J3) ; de présentations orales au groupe d'outils produits et de leur utilisation dans la pratique d'accompagnement (J3) ; et enfin, d'écrits individuels post-formation (J4 et juin 2016).

\section{3. Analyse à l'aide des indicateurs de développement professionnel des EA}

La première étape de l'analyse porte sur le développement professionnel des EA. Les indicateurs de ce développement professionnel, tant dans le domaine de la construction de compétences que dans celui de la construction identitaire d'un genre $E A$, se retrouvent dans les données sous les formes indiquées en 3.3 : nous effectuons une recherche de ces traces dans les données et analysons ensuite le processus de développement professionnel d'Elsa et de Thom. Nous procédons à une analyse croisée entre chercheurs, à la lumière des traces identifiées, puis à des discussions de clarification, avant de retourner une nouvelle fois vers les données pour raffiner l'analyse.

\section{4. Analyse des effets de la RAP}

La seconde étape de l'analyse concerne la caractérisation des liens entre le dispositif RAP, tel qu'il a été mis en œuvre et le processus de développement identifié chez les EA. L'activité « d'attribution et contribution » proposée dans le cadre d'une RAP (Chevalier et al., 2013, p.117) permet les rapprochements entre les effets, en matière de développement 


\section{eJRIEPS 45 (Avril 2019)}

professionnel et leurs causes. A l'issue de la dernière session, les participants sont amenés à se rappeler les objectifs, la durée et les activités précises qui y ont été effectuées (annexe 1). Ils identifient ensuite les changements observés dans leur pratique professionnelle. Enfin, ils lient finement ces résultats aux conditions et aux moyens de la formation, ce qui permet d'accéder à son degré d'efficience. Dans notre recherche, c'est la mise en synergie des potentiels de la RAP et des connaissances issues à la fois la recherche et de l'expérience des participants qui contribue à enclencher et accompagner un processus de développement professionnel. Nous travaillons en équipe de chercheurs pour dégager des liens plus ténus entre les présupposés des options théoriques et les choix méthodologiques précis par journée de formation et les effets réels sur le processus de développement professionnel des EA.

\section{Résultats}

A la lumière des indicateurs et des phases identifiées, nous présentons une analyse du processus de développement professionnel de deux EA du groupe : Elsa et Thom. Nous éclairons notre description d'exemples concrets issus des entretiens ou des moments de formation.

\subsection{Deux études de cas : Elsa et Thom}

Elsa occupe depuis 25 ans des fonctions, d'enseignante d'EPS au collège et au lycée, de formatrice d'enseignants et d'EA. Sa principale préoccupation en formation en EPS est que tous puissent se sentir bien avec leur corps, incluant leurs émotions et ressentis. Elle considère que l'accompagnement d'un stagiaire passe par « le questionnement chez la personne en formation », lequel est suscité par le biais d'échanges pour " chercher ensemble » (entretien pré-formation). Elle sort de la formation avec un renouvellement de ses connaissances sur sa pratique d'accompagnement : « il y a un avant et un après la formation » (écrit réflexif).

Thom enseigne l'EPS dans un lycée de Reims. Depuis quelques années, il occupe aussi la fonction d'EA auprès de stagiaires. II se dit solidement installé dans une pratique qu'il considère efficace. II se décrit comme un EA « interventionniste » (entretien pré-formation), s'appuyant sur 20 ans d'expérience en enseignement. Au cours de la RAP, il transformera sa posture d'enseignant-expert à accompagnateur.

5.1.1. Elsa, un métier d'EA « déjà là » en apprentissage continu Elsa s'engage dans la formation pour apprendre davantage sur le métier d'EA, selon ses propres mots. Elle dit se questionner constamment et avoir à cœur de s'améliorer. Bien 


\section{eJRIEPS 45 (Avril 2019)}

qu'elle soit motivée par la formation offerte, elle s'engage surtout curieuse de mieux connaitre le processus universitaire de formation. Au bilan du J1, elle ne semble pas convaincue par ce qu'elle pourrait y apprendre, bien qu'elle salue la dynamique de groupe qui s'est créée, par le truchement du travail chercheur-formateur. Elle mentionne se sentir « dans la contrainte » (contrainte par son horaire) pour la suite la formation. Elle s'ouvre toutefois à une possible déstabilisation, en acceptant l'entrée dans une phase d'insécurité. $\mathrm{Au}$ bilan du $\mathrm{J} 2$, de premières traces de réflexivité apparaissent, alors qu'elle partage la reconnaissance de « la densité des éléments [d'analyse de la pratique d'EA] qui apparaissent au fur et à mesure des échanges ». II est possible d'identifier chez elle deux situations de formation, l'entretien d'auto-confrontation croisée au J2 et le retour sur la roue socratique au J4 (fig. 3) qui induisent des apprentissages déterminants, tels que l'observation ciblée ou des transformations de posture dans le dilemme sur l'intervention ou non. Le groupe, incluant la posture du chercheur-formateur, constitue aussi une stimulation pour son apprentissage. Elle le partage à plusieurs reprises, notamment au bilan du J2, en s'adressant à lui : « je trouve que c'est un point positif dans le groupe, le guidage que tu mets ».

Chez elle, c'est au bilan du J3 que des traces d'apprentissages deviennent évidentes, sous la forme de la réflexivité, ainsi que de l'engagement : " je me sens mobilisée, interpellée » (bilan J3), lorsqu'elle parle de l'activité autour de sa vidéo en observant son étudiant. Ses pairs l'interpellent notamment sur le fait qu'elle en fait peut-être un peu trop pour lui, voire à sa place. On lui suggère même qu'elle n'oserait pas laisser le stagiaire se planter. Les échanges entre pairs l'amènent à se questionner sur les choix d'intervention lors de ses prochaines visites de supervision. "Est-ce que j'en fait trop ? " se dit-elle. Elsa est déstabilisée dans sa pratique d'EA. Elle pensait être peu interventionniste et reconnait se voir autrement, se rapprochant de «son opposé » (Thom), à qui elle s'adresse en se reconnaissant dans cette autre pratique :

... les morceaux de vidéo où on était sur des mêmes postures d'intervention et de fin de séance avec le stagiaire, je pense que d'un premier discours, je dirais que je ne sais pas trop pourquoi je fais quoi ? (...) dans les prochaines visites, il y a des choses que je ne ferai plus ou que je ferai quand même parce qu'elles n'ont pas d'incidence sur la construction du stagiaire dans la prise en charge de son groupe ou dans la mise en œuvre de son enseignement... (bilan J3).

À la fin de ce dernier extrait, apparaissent également des traces de généralisation de son activité d'EA, lorsqu'elle commente sur ses interventions futures (ce qu'elle fera ou pas) 


\section{eJRIEPS 45 (Avril 2019)}

pour contribuer à la construction des compétences du stagiaire. Elle s'approprie également au même moment des outils proposés dans la RAP. L'entretien d'auto-confrontation (J2) soutient son développement professionnel, qu'elle voit comme un engagement dans un questionnement sur son activité d'EA. Elle dit en arriver là parce qu'elle se sent en sécurité dans le groupe de collègues, ce qui lui permet de rencontrer les autres et de confronter ses idées :

... il y a eu un avant et un après la vidéo. J'ai été mise en face de moi-même et puis obligée de répondre à des questions : Qu'est-ce que tu lui as dit ? Qu'est-ce qu'il t'a répondu ? Pourquoi tu lui as demandé ça ? Pourquoi t'as fait ça ? Je reconnais que des fois parce que je n'en avais pas envie, que je ne savais pas ou que j'étais agacée par la question, j'avais pas forcément envie d'expliquer aux autres pourquoi j'ai fait ça. Cela n'a pas été une confrontation simple. Dans mes visites après, il y avait toujours un petit peu dans un coin du gymnase l'ombre de C.(chercheur-formateur) et de mes partenaires du groupe. Les choses ont été éclairées différemment... (J4 : activité retour sur la roue socratique).

$\mathrm{Au} \mathrm{J4}$, elle témoigne de son apprentissage dans la formation et de sa contribution à la recherche, en lien avec son souhait initial :

... je trouve que c'est ce que tu nous as offert [au chercheur-formateur], la possibilité d'être confrontés à d'autres connaissances, façons de faire, personnes et dans d'autres fonctionnements. Puis du coup, de connaître un petit peu mieux ce que c'est qu'un travail de recherche et de pouvoir y contribuer (...) le travail d'EA n'est pas abouti, on le voit au travers de la roue. J'aimerais un stagiaire l'année prochaine, pour faire vivre autrement, parce que cette année, j'ai essayé de le faire en route mais c'était aussi dans la formation... (bilan J4).

Elle n'en reste pas là. Son travail sur sa roue socratique montre une évaluation fine de l'atteinte de ses objectifs (fig. 3., ligne violette). Lorsqu'elle réévalue ses compétences identifiées en début de formation (ligne bleue), elle les réajuste aussi légèrement à la baisse (ligne rose). Ceci lui permet de témoigner d'une amélioration de ses compétences (ligne verte). Déjà, Elsa annonce ses intentions de s'engager dans la poursuite de son développement professionnel. Si ces éléments présentent des effets perçus en fin de formation, une information ultérieure de la part de Elsa sous la forme d'un SMS envoyé au chercheur-formateur en début d'année suivante montre un effet réel et une consolidation de la transformation, sur le thème de l'interventionnisme d'un $E A$ : « je pense à toi ! Je ne 


\section{eJRIEPS 45 (Avril 2019)}

ramasse aucun ballon. Je suis vissée sur ma chaise ! ». Elle consolide également sa conscience de deux genres professionnels déjà présents en début de RAP : « le métier d'enseignant et le métier d'EA sont deux métiers totalement différents ! » (écrit postformation).

\subsubsection{Thom, la découverte d'un métier d'EA}

Bien qu'il ait émis des souhaits initiaux pour son développement professionnel, par exemple d'améliorer la qualité de ses relations avec les stagiaires, Thom se questionne sur la pertinence de la formation et les possibilités d'un apprentissage utile pour lui en tant qu'EA Ses premiers commentaires en témoignent : « mon engagement dans cette formation ne s'inscrit pas au départ dans une volonté de formation professionnelle » (entretien préformation) ; « je n'arrive pas encore à bien visualiser concrètement ce que ça va changer dans ma fonction d'EA » (bilan J1). II y a, au départ, un équilibre stabilisé dans sa pratique d'EA, très accrochée à sa pratique enseignante experte.

Au cours de la RAP, Thom décide de s'engager dans une phase d'expérimentation (J2J3), lorsqu'il offre de se laisser filmer en situation d'observation d'un étudiant pour répondre à un besoin de formation exprimé par le groupe de travailler sur des exemples concrets de pratique tirés de leur quotidien d'EA. Puis il pousse l'investissement en intégrant de nouveaux outils à sa pratique d'EA : il construit ses propres grilles d'analyse pour l'observation d'un stagiaire, ainsi qu'une roue socratique représentant les dimensions des tâches d'enseignement en EPS à évaluer chez le stagiaire (fig. 1), qu'il présente et discute avec le groupe (J4). Le travail en EACC (J2 et J3) est un passage fondamental dans la déstabilisation de ses convictions initiales plutôt modélisantes d'EA. Peu à peu, il développe sa réflexivité sur son activité d'EA, en questionnant le sens, des visées, des moyens et des résultats de son activité d'accompagnement. II commence à " se voir » intervenir « à la place » du stagiaire et repérer les effets sur ce dernier. Ainsi, lors d'un échange avec Elsa (dans un temps d'EACC), il s'interroge : « j'interviens beaucoup, peutêtre trop ? » en adressant sa question aux collègues en formation. II se demande ensuite (Bilan J3), si « un travail d'écoute » ne devrait pas prendre plus de place dans son travail d'EA. Ainsi, nous repérons une phase d'appropriation d'un genre EA dans son processus de développement entre J2 et J4, qu'il décrit ainsi :

... par rapport à la difficulté à se positionner, en tant qu'enseignant et EA, je trouve que l'aide du chercheur-formateur et du groupe nous a apporté pas mal de choses et finalement, je vais partir avec ma casquette d'EA. J'ai réussi à mettre de côté un peu l'enseignant d'EPS... (bilan J2). 


\section{eJRIEPS 45 (Avril 2019)}

Au terme du J3, il met à distance sa pratique par un mouvement de généralisation :

Je ne sais pas si on peut parler de mouvement de « subjectivation »? J'arrive à prendre un petit peu de distance avec ma personnalité, qui est peut-être un peu plus « rentre-dedans », c'est à dire d'aller dans mon sens. J'arrive à faire un petit pas de côté en me disant, qu'il (le stagiaire) prend le chemin qu'il veut, l'important c'est qu'il arrive à se former en tant qu'étudiant et que je sois là pour l'aider... (bilan J3).

Cette appropriation est partagée avec ses collègues, en rapprochant ses représentations de son rôle de celles d'Elsa (dans un temps d'EACC), notamment, lorsqu'il dit : “ j'ai exactement le même problème, c'est à dire, comment avec le savoir d'EA que t'as, transmettre et faire évoluer la personne qui est à tes côtés, ça c'est vachement dur ». Thom va même jusqu'à dire que la RAP a « radicalement transformé » la représentation qu'il se fait du rôle d'EA de celle d'enseignant-expert interventionniste à celle d'accompagnateur de l'apprentissage du stagiaire (bilan J4).

...j'ai l'impression que c'est un autre métier (EA), ça demande des compétences autres. En faisant ces journées, on arrive bien à identifier les compétences nécessaires. Puis, il y a aussi un positionnement, c'est-à-dire être capable de se détacher affectivement de son rôle d'enseignant et de se repositionner comme un EA... (bilan J3).

Au terme de la RAP, il adopte une posture de secondarité (Paul, 2009), «plus humaine » selon ses dires, centrée sur le stagiaire et la mise en valeur de son activité plutôt que sur la réussite de la tâche d'enseignement. Dans l'entretien pré-formation, Thom avait émis le souhait « d'apprendre à mieux tenir compte de la sensibilité et de la personnalité du stagiaire ». Ses questions sur son interventionnisme excessif et la réponse qu'il se donne sous la forme de "plus d'écoute ? » indiquent, pour le moins, une intention en ce sens. Enfin, son exercice de roue socratique de fin de formation (fig.2) montre qu'il auto-évalue ses compétences avec davantage de force (ligne rose grasse) qu'en début de formation (ligne rose fine) et surtout qu'il réactualise à la baisse (ligne rouge) sa position de départ, s'il devait se réévaluer au $\mathrm{J} 1$ : « je m'évalue moins bien aujourd'hui que lors de la première séance, parce que j'ai l'impression de m'être transformé » (bilan J4). Il va jusqu'à se projeter dans ses futures interventions comme EA :

...être moins normatif, directif, descendant mais plutôt ciblé sur des aspects. L'observation ciblée, ça me parle beaucoup. Je pense que c'est des choses que je vais mettre en place assez vite, essayer de ressortir des éléments qui me paraissent importants, par rapport à la personnalité de l'étudiant stagiaire, par rapport à son 


\section{eJRIEPS 45 (Avril 2019)}

mode de fonctionnement pour le transformer progressivement, enfin pour l'aider à se transformer progressivement... (bilan J4).

Une phase de consolidation du développement professionnel de Thom suit puisque, l'année suivante, il s'inscrit dans le processus de certification à la fonction d'EA (CAFFA : Certificat d'Aptitude aux Fonctions de Formateur Académique).

Nous repérons ainsi du développement, de l'approfondissement ou des transformations de compétences pour les deux EA ainsi que des évolutions du point de vue identitaire quant à la différenciation des fonctions d'enseignant et d'EA. Nous poursuivons l'analyse sur la question de ce qui peut être attribué à ces déplacements, dans cette RAP.

5. 2. Attribution-contribution (RAP-développement professionnel) pour Elsa et Thom Nous relevons différents éléments jouant un rôle dans le processus de développement professionnel des EA. L'utilisation d'un outil de transformation et d'analyse de l'activité associé à des apports de connaissances, issues de la recherche et de l'expérience ont permis aux EA d'effectuer un déplacement de point de vue sur leur pratique et d'ainsi construire des apprentissages. Pour Elsa comme pour Thom, un moment fort de la formation se situe entre le J2 et le J3, avec un point culminant lors des EACC. Se regarder et développer explicitement ses choix, ses buts et les justifier, transforme cette activité. Bénéficier du retour des pairs et du chercheur-formateur pour questionner, discuter et débattre et conceptualiser les buts et les choix au regard de son activité pousse davantage l'analyse. Cela permet de comprendre, sans juger, en repérant des effets sur le débutant ou sur les élèves. Les EA envisagent des pans de leur activité qu'ils ne soupçonnaient pas et prennent conscience plus objectivement de leur pouvoir d'agir.

Par ailleurs, il y a lieu de croire que le parti pris « collaboratif » de la RAP favorise la qualité de ces moment forts de développement professionnel. Nous l'observons dans sa mise en action au J1 et J2, par le choix de l'objet de formation en groupe et par une préparation des participants du point de vue de la relation de confiance entre les EA participants, puis dans les régulations de $\mathrm{J} 3$ et $\mathrm{J} 4$, ainsi que dans le souci de lier l'expérience des EA aux connaissances et outils issus de la recherche, apportés par le chercheurformateur. Les deux EA mentionnent, dans leurs partages oraux, qu'ils ne sont pas habitués à une formation de ce type, qui leur donne peu de cadrage préétabli mais laisse une liberté de choix accompagnés, qui met en valeur leur pratique pour la faire évoluer et non en proposant des choix définis à l'avance. Au J1 et encore au J2, les participants demandent 


\section{eJRIEPS 45 (Avril 2019)}

du cadrage de la part du chercheur-formateur et disent ne pas bien savoir où ils vont (Thom) ; malgré cette incertitude, tous deux s'engagent dans les activités proposées.

Le type et la variété des dispositifs de formation sont des éléments essentiels au maintien de la motivation et à l'engagement des EA, en lien avec la pertinence des transformations qu'ils perçoivent. L'apport de la recherche (outils et connaissances) est également perçu comme structurant et nécessaire pour aller plus loin dans la stabilisation des compétences ou postures nouvelles (cf. extraits de verbatim d'Elsa p.12-13 et de Thom p.14). Enfin, la dynamique du collectif, les échanges et mutualisation entre pairs sont identifiés comme fondamentaux pour se reconnaitre dans cette fonction, se donner des repères dont les EA disent avoir besoin pour se sentir légitimes et que l'institution ne leur donne pas. Ces éléments identifiés dans les écrits réflexifs mettent en avant l'intérêt et la pertinence de la RAP dans le processus de développement professionnel.

\section{Discussion}

L'analyse du processus de développement professionnel d'Elsa et de Thom, dans le cadre d'une RAP, permet d'identifier des avantages et des limites. Elle ouvre en outre sur une discussion plus large dépassant le contexte français, à propos d'un scénario possible de formation des EA à l'accompagnement d'un débutant.

\section{1. Avantages et limites}

A la lumière de nos résultats, nous constatons que la RAP, dans sa dimension formation, a des effets positifs sur le processus de développement professionnel d'Elsa et de Thom. La dimension recherche de la RAP apporte par ailleurs une rigueur au processus de formation qui permet aux EA de «se voir » avec davantage de recul dans leur activité, en se positionnant dans un contexte plus large, généré entre autre par le cadre international du projet de recherche et la visée partagée de production de connaissances et de communication sur l'objet. Dans le contexte français, l'approche RAP tend à se différencier des modèles utilisés en formation continuée, plus cadrants dans la définition de l'objet de formation et dans la régulation en cours de dispositif. Cette approche différente de modèles plus connus, notamment par la co-construction des objets de formation tout au long du parcours, a l'avantage de créer un effet de curiosité chez les participants, induisant une remise en question, voire une déstabilisation temporaire de leurs repères dans leur activité habituelle en formation. Elsa et Thom saisissent cette opportunité pour prendre en charge leur apprentissage, notamment par des prises de risques dans leur pratique d'accompagnement. Ils en font ainsi bénéficier le groupe dans les moments communs 


\section{eJRIEPS 45 (Avril 2019)}

d'analyse, qui en retour, leur apporte une ressource essentielle de développement par la richesse de l'échange en toute confiance. Leur engagement a un effet sur la dynamique du groupe. Les cinq participants entrent dans des négociations de construction d'un genre professionnel d'EA, vers un modèle de pratique d'accompagnement moins interventionniste que celui observé jusqu'à ce jour.

En revanche, les EA qui n'ont pas charge d'accompagnement entre les sessions semblent freinés dans leur processus de développement professionnel, particulièrement dans la phase d'appropriation, même s'ils demeurent dans la dynamique de travail du groupe. Eric dit : «mon seul regret est de ne pas avoir pu expérimenter une partie du travail et de le partager avec les membres du groupe », même s'il perçoit des apprentissages tels que « le début de construction d'un cadre d'observation par la catégorisation et l'explicitation de certains critères et un début de transformation dans la façon d'observer et d'intervenir [s'il avait de nouveau à accompagner], ainsi que de hiérarchiser les priorités » (écrit postformation).

6. 2. Ouverture sur un scénario de formation à l'accompagnement Cette formation en contexte français mène à penser qu'une séquence de journées de RAP en lien avec une pratique d'accompagnement sur le terrain professionnel permet aux EA de conduire le processus de développement professionnel de façon probante dans les phases identifiées (Boucher et Jenkins, 2004). Cette construction s'effectue dans la mesure où cette pratique est à la fois source et matière du travail mené en présentiel avec le groupe. Par ailleurs, nous identifions que les connaissances et les outils issus de la recherche sont essentiels à la structuration des compétences développées par les participants. Ils contribuent à la nouvelle activité d'accompagnement mise en œuvre avec les débutants dans un temps suffisant d'appropriation. La présence des pairs et la confiance vécue dans le groupe permet de tolérer l'incertitude, de se laisser déstabiliser, de prendre des risques dans sa propre pratique d'accompagnement, sachant qu'une discussion dans le groupe peut ensuite aider à aller plus loin et à stabiliser des choix ou à les revoir. Pour arriver à une consolidation, il semble toutefois intéressant d'augmenter le temps de ce dispositif au moins sur deux années consécutives. Ceci permettrait d'observer les transformations et d'aborder d'autres objets de formation et compétences liées à la fonction, dans la même dynamique de travail. Par ailleurs, multiplier ces RAP dans les plans de formation est une option qui nous parait pertinente.

\section{Conclusion}




\section{eJRIEPS 45 (Avril 2019)}

$\mathrm{Au}$ terme de ce travail, nous pouvons dire que les EA, en s'engageant dans un collectif professionnel apprenant en RAP, tel que proposé dans le contexte français, ont construit des compétences dans leur pratique d'accompagnement d'un enseignant débutant en EPS. Ils l'ont fait par le biais d'une pratique et d'une réflexion sur la pratique. Par ailleurs, la construction d'un genre professionnel spécifique d'EA est identifiée, complémentaire à ce travail sur les compétences liées à la fonction. Pour Elsa, c'est un renforcement d'une conscience déjà construite d'effectuer deux métiers différents intimement liés : EA et enseignant. Pour Thom, c'est une découverte et une entrée dans une nouvelle identité professionnelle, double. Des phases de développement sont repérables dans le temps de la RAP et même au-delà de celle-ci, témoignant d'un processus en marche. Le choix d'une RAP contribue à l'engagement et à l'apprentissage des EA en s'appuyant sur leur pouvoir d'agir. Ceux-ci, dans une dynamique collaborative et un climat de confiance, démontrent leur capacité de réflexion approfondie, lorsqu'elle est déclenchée par des outils de transformation et d'analyse de leur activité, soutenue dans sa structuration par des connaissances issues de la recherche et de l'expérience. La RAP présente elle-même des dimensions propres à une pratique d'accompagnement (Paul, 2009) : une posture de secondarité du chercheur-formateur et de l'équipe de recherche ; un cheminement régulé avec les EA dans un espace-temps de transition connu ; et un véritable effet d'ensemble, qui au-delà d'un changement d'état des EA, ne laisse pas le chercheur-formateur indemne. Ainsi, le processus de développement professionnel du chercheur-formateur dans une RAP sera également intéressant à caractériser et fera l'objet d'un article ultérieur.

\section{Bibliographie}

Altet, M., Paquay, L., et Perrenoud, P. (2002). Formateurs d'enseignants : quelle professionnalisation ? Bruxelles : De Boeck.

Boucher, L.-P. et Jenkins, S. (2004). Un soutien aux processus de transformation des pratiques au primaire. In M. L'Hostie et P. Boucher (Eds), L'accompagnement en éducation. Un soutien au renouvellement des pratiques (pp. 84-106). Québec: Presses de l'Université du Québec.

Boudreau, P. (2009). Pour un modèle de supervision de type inductif en formation des maîtres de stage en éducation physique. Éducation et francophonie, 37(1), 121 139. 


\section{eJRIEPS 45 (Avril 2019)}

Boutet, M. et Villemin, R. (2014). L'accompagnement : un élément clé pour l'apprentissage en stage et pour le développement professionnel continu des enseignants. Phronesis. 3 (1-2). 81-89.

Boutet, M. et Pharand, J. (2008). L’accompagnement concerté des stagiaires en enseignement. Québec : PUQ.

Bucheton, D. et Soulé, Y.(2009), « Les gestes professionnels et le jeu des postures de l'enseignant dans la classe : un multi-agenda de préoccupations enchâssées », Éducation et didactique [En ligne], vol 3 - n’3 / Octobre 2009, mis en ligne le 01 octobre 2011.

Carlier, G. (2009). Accompagner et former des maîtres de stage en éducation physique. L'expérience de l'Université catholique de Louvain (Belgique). Éducation et Francophonie, 37 (1), 68-88.

Chaliès, S. et Durand, M. (2000). L'utilité discutée du tutorat en formation initiale des enseignants. Recherche et Formation. 35, Paris : INRP.

Chevalier, J. et Buckles, D. (2013). Participatory Action Research. Theory and Methods for Engaged Inquiry. New-York: Routledge.

Chevalier, J., Buckles, D. et Bourassa, M. (2013). Guide de la recherche-action, la planification et l'évaluation participatives, SAS2 Dialogue, Mars, Ottawa (Canada).

Clot, Y. (1999). La fonction psychologique du travail. Paris: Presses universitaires de France.

Desbiens, J.-F., Borges, C. et Spallanzani, C. (2009). Investir dans la formation des personnes enseignantes-associées pour faire du stage en enseignement un instrument de développement professionnel. Éducation et francophonie, 37(1), 625.

Desbiens, JF., Spallanzani, C. et Borges, C. (Eds) (2013). Quand le stage en enseignement déraille : regards pluriels sur une réalité trop souvent occultée. Québec : PUQ.

Dewey, J. (1949, 1938). Experience and Education. New-York : MacMillan.

Donnay, J. (2008). Préface. In M. Boutet \& J. Pharand (dir.), L'accompagnement concerté des stagiaires en enseignement. Québec : Presses de l'Université du Québec.

Grosstephan, V., (2010). Développement professionnel d'enseignants d'EPS : processus et effets dans le cadre d'un dispositif de formation continue associant praticiens et chercheur. Thèse de doctorat non publiée. Université de Reims ChampagneArdenne. 


\section{eJRIEPS 45 (Avril 2019)}

Jorro, A., (2014). Développement professionnel. In Jorro, A. (dir.). Dictionnaire des concepts de la professionnalisation. Bruxelles : De Boeck. 75-79.

Lefeuvre, G., Garcia, A., \& Namolovan, L., (2009) « Les indicateurs de développement professionnel », Questions Vives [En ligne], Vol.5 n¹1|2009, mis en ligne le 01 juin 2011, consulté le 21 septembre 2016. URL: http://questionsvives.revues.org/627 ; DOI : 10.4000/questionsvives.627

Matteï-Mieusset, (2013). Les dilemmes d'une pratique d'accompagnement et de conseil en formation. Analyse de l'activité réelle du maître de stage dans l'enseignement secondaire. Thèse de doctorat, non publiée. Université de Reims ChampagneArdenne.

Mattéï-Mieusset, C., Brau-Antony, S. (2016). Les dilemmes d'une pratique d'accompagnement en formation d'enseignants. Analyse de l'activité réelle du maître de stage. Revue des sciences de l'éducation, Volume 42, Numéro 2, 2016 , p. 149-173.

Molina, C. E. et Gervais, C. (2008). Les stages en formation à l'enseignement : pratiques et perspectives théoriques. Québec: Presses de l'Université du Québec.

Paul, M. (2009). Autour du mot « accompagnement ». Recherche et Formation, 62, 91-107. Rogers, C. (1970). On Encounter Groups. New-York : Narrow.

Roquet, P. (2009). L'émergence de l'accompagnement: une nouvelle dimension de la formation. Recherche et Formation, 62, 13-24.

St-Arnaud, Y. (2003). L'interaction professionnelle : efficacité et coopération. Montréal : PUM.

\section{Annexes}

Annexe 1

\section{Consignes pour l'écrit réflexif post-RAP}

Points sur lesquels vous pensez avoir ou pas engagé des transformations ?

- Engagement dans un dispositif de formation/recherche «type co-construction de la formation »

- Impact sur le métier d'enseignant d'EPS.

- Impact sur la fonction d'EA.

- Liens perçus entre le métier d'enseignant et la fonction d'EA.

- Collectif du groupe de formation et expériences mutualisées. 
eJRIEPS 45 (Avril 2019)

- Rapport aux connaissances théoriques.

A quels éléments du dispositif attribuez-vous ces transformations (ou non) ?

- Libre

- $\quad$ Si suite de formation, quelles perspectives ? Quels objectifs ? 